Sādhanā, Vol. 19, Part 5, October 1994, pp. 751-764. (C) Printed in India.

\title{
Natural convection of a radiating fluid in a square enclosure with perfectly conducting end walls
}

\author{
A YÜCEL, S ACHARYA* and M L WILLIAMS \\ Louisiana State University, Baton Rouge, LA 70803, USA
}

\begin{abstract}
Combined natural convection and radiation in an asymmetrically heated square enclosure is studied numerically with both adiabatic and perfectly conducting end walls. The momentum and energy equations are solved by a control volume based finite difference algorithm which is coupled with the discrete ordinates method for radiative heat transfer calculations. The changes in the flow patterns and temperature distributions due to the presence of radiation in an enclosure with conducting end walls are compared with those for the case of an enclosure with adiabatic end walls, and significant differences are noted. The flow field is stronger, and the heat input along the hot wall and the end walls are greater for the conducting end wall case. The effects of optical thickness, scattering and wall emissivity on the flow and temperature fields and heat transfer rates are analysed.
\end{abstract}

Keywords. Natural convection; radiation; enclosure.

\section{Introduction}

Thermal radiation can strongly interact with enclosure natural convection in many situations of engineering interest such as nuclear reactor safety, combustion, fire and plumes, porous media and solar collectors. Ostrach (1988) provides a comprehensive review of studies where natural convection is the only mode of heat transfer in enclosures. Studies dealing with the interaction between radiation and natural convection has been the subject of reviews by Viskanta (1984), Yang (1986) and Viskanta \& Menguc (1987). Representative studies include those reported by Lauriat $(1982 \mathrm{a}, \mathrm{b})$ who used the $P-1$ differential approximation to study radiation-convection interactions of a participating medium in vertical enclosures. Desrayaud \& Lauriat (1985) later extended the study for a radiating vertical fluid layer. Using the $P-1$ approach, Fusegi \& Farouk (1986, pp. 81-8) studied the interaction in a square enclosure with asymmetrical heating. They later extended the study for fire-spread applications (Fusegi \& Farouk 1987, pp. 63-8). Webb \& Viskanta (1987) studied the

\footnotetext{
* For correspondence
} 
natural convection caused by the irradiation of a fluid layer. Yücel et al (1989) used the discrete ordinates method to study the combined natural convection-radiation interactions in an enclosure with a participating medium. Kassemi \& Duval (1990) employed a zonal approach to analyse the effects of radiation on the transport process in rectangular enclosures. Kassemi \& Naraghi (1990) used a discrete exchange factor method described by Naraghi \& Kassemi (1989) to study radiation-natural convection interactions in a square enclosure. Tan \& Howell (1991) used the product integration method (PIM) proposed by Tan (1989) together with a nonlinear-SOR strategy to study radiation-natural convection in a square enclosure.

In the above-mentioned studies of natural convection-radiation interactions in asymmetrically heated enclosures, adiabatic conditions are customarily assumed on the end walls. Yet, adiabatic conditions are not easily realized in experimental settings or engineering applications. The effects of conducting end walls should be taken into consideration for more realistic simulations. In this paper, two-dimensional natural convection of a radiating fluid in an externally heated vertical square enclosure is studied. The end walls are assumed to be perfectly conducting, and results are compared with results obtained with the assumption of adiabatic end walls. The perfectly conducting end wall condition has been shown to be a realistic end wall condition, with gas as the working fluid (ElSherbiny et al 1982) and represents a first approximation to account for the influence of conduction along the enclosure walls.

Various solution methods are currently available for the solution of radiative fluxes. These include: the zonal method, the flux method, the ray-tracing method, the Monte-Carlo method, the discrete exchange factor method, the product integration method, the finite volume method and the discrete ordinates method. More details on many of these methods can be found in Viskanta (1984), and Viskanta \& Menguc (1987). In this paper, the discrete ordinates method is used to solve the radiation part of the problem. In comparison with many other competing methods, the discrete ordinates method has been shown to yield higher order accurate solutions to the equations of radiative heat transfer in problems involving radiation affected buoyant enclosure flows (Yücel et al 1989). In the present work, the radiation calculations using the discrete ordinates method are coupled with a control volume based finite difference algorithm which is used to solve the momentum and energy equations. The changes in the natural convection flow patterns and temperature and heat transfer distributions due to the presence of radiation are analysed and results compared for both linear temperature and adiabatic boundary conditions.

\section{Analysis}

The physical model consists of a gray, absorbing, emitting and isotropically scattering fluid in a square enclosure (figure 1). The fluid is Newtonian and incompressible; viscous dissipation is neglected and physical properties are taken as constant except for the density. The flow is assumed to be laminar, steady and two-dimensional. With these assumptions, the equations for the conservation of mass, momentum and energy can be expressed in dimensionless form as follows.

$$
\frac{\partial U}{\partial X}+\frac{\partial V}{\partial Y}=0
$$




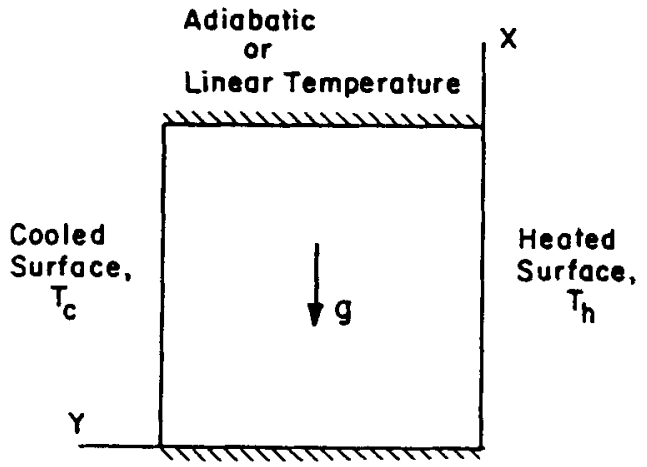

Figure 1. Schematic representation of the physical system.

$$
\begin{aligned}
& U \frac{\partial U}{\partial X}+V \frac{\partial U}{\partial Y}=-\frac{\partial P}{\partial X}+\nabla^{2} U+\frac{\operatorname{Ra}_{\mathbf{E}}}{\operatorname{Pr}} \phi \\
& U \frac{\partial V}{\partial X}+V \frac{\partial V}{\partial Y}=-\frac{\partial P}{\partial Y}+\nabla^{2} V \\
& U \frac{\partial \phi}{\partial X}+V \frac{\partial \phi}{\partial Y}=\frac{1}{\operatorname{Pr}} \nabla^{2} \phi-\frac{\phi_{0}}{\operatorname{Pl} \operatorname{Pr}} \nabla \cdot \mathbf{Q}_{R}
\end{aligned}
$$

where the Boussinesq approximation $\left(\rho=\rho_{0}\left[1-\beta\left(T-T_{0}\right)\right]\right)$ was used in the buoyancy term to allow for the variation of density with temperature. In the above equations, the buoyancy induced by external heating is represented by the external Rayleigh number $\mathrm{Ra}_{E}$.

The term $\left(\phi_{0} / \mathrm{Pl} \cdot \operatorname{Pr}\right) \nabla \cdot \mathbf{Q}_{R}$ in the energy equation represents the radiative energy deposition and is determined from the solution of the radiative transfer equation. The equation governing the steady state dimensionless radiation intensity $I$ is given by

$$
\mu(\partial I / \partial X)+\xi(\partial I / \partial Y)+\tau I=(\tau / 4 \pi)\left[(1-\omega) B+\omega \int_{4 \pi} I \mathrm{~d} \Omega\right]
$$

where $I$ is the dimensionless intensity of radiation at a point $(X, Y)$ in the direction $\mathbf{\Omega}$. The emissive power $\boldsymbol{B}$ is expressed as

$$
B=\left(T / T_{0}\right)^{4}=\left(\phi / \phi_{0}+1\right)^{4} .
$$

The radiative heat flux vector can be calculated by the intensity distribution,

$$
\mathbf{Q}_{R}=\int_{4 \pi} \Omega I \mathrm{~d} \Omega=\int_{4 \pi}(\mu \mathbf{i}+\xi \mathbf{j}) I \mathrm{~d} \Omega .
$$

Integrating (5) over the whole solid angle yields a relation for the divergence of the radiative heat flux vector

$$
\nabla \cdot \mathbf{Q}_{R}=(1-\omega) \tau\left[B-\int_{4 \pi} I \mathrm{~d} \Omega\right]
$$

which is substituted in the energy equation. 


\subsection{Boundary conditions}

No-slip (zero velocity) boundary conditions are imposed on the rigid walls. Two end-wall boundary conditions are considered for temperature: either thermally insulated (adiabatic case) or perfectly conducting (linear temperature case). These conditions along $X=0$ and $X=1$ are expressed as:

$$
\begin{array}{ll}
\text { adiabatic case: } & \mathbf{Q}_{T} \cdot \mathbf{i}=\left(-\nabla \phi+\left(\phi_{0} / \mathrm{Pl}\right) \mathbf{Q}_{R}\right) \cdot \mathbf{i}=0, \\
\text { linear temperature case: } & \phi(X, Y)=0 \cdot 5-Y .
\end{array}
$$

The two other walls are maintained at temperatures $T_{h}$ (hot wall) and $T_{c}$ (cold wall) respectively. For black surfaces the intensity of radiation leaving a bounding surface is simply the blackbody intensity of radiation emitted at the surface temperature. For diffusely reflecting and emitting end walls, the boundary intensity in the direction $\boldsymbol{\Omega}$ leaving the surface is given by

$$
\begin{array}{r}
I(X, Y, \Omega)=\frac{\varepsilon B(X, Y)}{4 \pi}+\frac{(1-\varepsilon)}{\pi}\left[\int_{\mathbf{n} \cdot \mathbf{\Omega},<0}|\mathbf{n} \cdot \Omega| I\left(X, Y, \Omega^{\prime},\right) \mathrm{d} \Omega^{\prime}\right], \text { at } \\
X=0 \text { and } X=1,
\end{array}
$$

where $\varepsilon$ is the surface emissivity ( $\varepsilon=1$ for black surfaces) and $n$ is the unit normal at the surface.

\subsection{Solution procedure}

Equations (1) $(8)$ with the associated boundary conditions provide a complete mathematical formulation of the problem. The mass, momentum and energy equations are solved by a control-volume based finite difference procedure. Pressure-velocity interlinkage is resolved using the SIMPLER algorithm (Semi Implicit Method for Pressure Linked Equations Revised). This method is described in detail by Patankar (1980). The solutions to the equation of radiative transfer are obtained by the discrete ordinates method. In the discrete ordinates formulation, the solid angle is discretized by selecting an ordered set of directions with predetermined weights. Quantities involving integrals over the solid angle are evaluated by sums over the discrete directions with weights as the weighting functions. The spatial discretization introduced to obtain a finite difference form of the radiative transfer equation employs the same control volumes (cells) as the SIMPLER algorithm. For example, for a control volume bounded by $\left(X_{i}, X_{i+1}\right)$ and $\left(Y_{j}, Y_{j+1}\right)$, the discretized form of (5) can be expressed as

$$
\mu_{m} \frac{\left(I_{i+1}-I_{i}\right)}{\left(X_{i+1}-X_{i}\right)}+\xi_{m} \frac{\left(I_{j+1}-I_{j}\right)}{\left(Y_{j+1}-Y_{j}\right)}+\tau I=S,
$$

where $I$ is the intensity at the centre of the control volume, and $\mu_{m}$ and $\xi_{m}$ are the direction cosines of the vector $\Omega$. The above equation represents the radiative energy balance for a control volume, with the first two terms on the left-hand side of the 
equation representing the loss of radiant energy across the control volume boundaries, the third term representing the loss by absorption and scattering, and the source term on the right representing the gains due to inscatter from other directions and due to emission.

The interface and cell-centre $I$-values are related using the linear or the step interpolation scheme which, in the $i$ - and $j$-directions, are mathematically expressed as

$$
I=a I_{i+1}+(1-a) I_{i}=a I_{j+1}+(1-a) I_{j}
$$

with $a=0.5$ for the linear model and $a=1$ in the step model. Equations (11) and (12) together lead to the discrete ordinates equation, which is solved by sweeping away from the boundaries. In the presence of scattering media or reflecting boundaries, the discrete ordinates equations are coupled and therefore have to be solved iteratively. Details of the discrete ordinates methodology can be found in Duderstadt \& Martin (1979), Fiveland (1987, pp. 9-18) and Yücel et al (1989).

The coupled mass, momentum, energy and radiative transfer equations are solved through a global iterative procedure which is outlined below:

(1) Initial velocity and temperature fields are assumed.

(2) The radiative transfer equation is solved for the given temperature field. The incident radiant energy term in (8) and the boundary radiative heat flux terms in (9a) are then determined.

(3) Equations (1)-(4) are solved to obtain a new flow field and temperature distribution.

(4) The next iteration is performed by repeating steps 2 and 3 . The procedure is continued until convergence is obtained on velocity and temperature to within a prespecified tolerance $\left(\left|\phi_{i j}^{n+1}-\phi_{i j}^{n}\right| /\left|\phi_{i j, \max }^{n}\right| \leqslant 10^{-5}\right)$.

\subsection{Numerical uncertainty}

The calculations are performed on a $32 \times 32$ nonuniform grid packed towards the walls (generated by an algebraic stretching procedure with a packing factor of 1.5 ) which was found to be adequate for natural convection in externally heated vertical and inclined enclosures (Acharya \& Goldstein 1985; Yücel et al 1989). Table 1 provides a comparison, for two grid sizes, of the values of the dimensionless total and radiative heat fluxes along the hot wall for the adiabatic end wall case. It can be seen that the two solutions agree to within $1 \%$ of each other. Furthermore, the overall energy

Table 1. Dimensionless average heat fluxes on the hot wall for the adiabatic end case, $\mathrm{Ra}_{\mathrm{E}}=5 \times 10^{6}, \mathrm{Pl}=0.02, \omega=0$.

\begin{tabular}{lccccc}
\hline & \multicolumn{2}{c}{$32 \times 32$ Grid } & & \multicolumn{2}{c}{$50 \times 50$ Grid } \\
\cline { 2 - 3 } \cline { 6 - 7 }$\tau$ & Total, $\bar{Q}_{T}$ & $\begin{array}{c}\text { Radiation } \\
\bar{Q}_{R} \phi_{0} / \mathrm{Pl}\end{array}$ & & Total, $\bar{Q}_{T}$ & $\begin{array}{c}\text { Radiation } \\
\bar{Q}_{R} \phi_{0} / \mathrm{Pl}\end{array}$ \\
\hline Non-radiating & 13.83 & 0 & & 13.76 & 0 \\
5.0 & 31.93 & 23.77 & & 31.76 & 23.64 \\
1.0 & 39.36 & 31.63 & & 39.21 & 31.55 \\
0.2 & 46.45 & 37.67 & & 46.11 & 37.40 \\
\hline
\end{tabular}


balance requires

$$
\begin{aligned}
& R=\int_{x=0}^{1}\left[\left(\mathbf{Q}_{T} \cdot \mathbf{j}\right)_{Y=0}-\left(\mathbf{Q}_{T} \cdot \mathbf{j}\right)_{Y=1}\right] \mathrm{d} X \\
&+\int_{X=0}^{1}\left[\left(\mathbf{Q}_{T} \cdot \mathbf{i}\right)_{X=0}-\left(\mathbf{Q}_{T} \cdot \mathbf{i}\right)_{X=1}\right] \mathrm{d} Y=0 .
\end{aligned}
$$

In all cases presented here, $E$ differs from zero in only the third digit after the decimal point.

\section{Results and discussion}

In this section results are presented in the form of streamline and isotherm contour plots, midplane velocity profiles and heat transfer rates on the walls. The dimensionless stream function is obtained from the velocity field solution by evaluating the integral

$$
\psi=\int_{0}^{1} U d Y,
$$

along constart $X$ lines with $\psi=0$ at $X=Y=0$.

Numerical solutions are obtained for a Prandtl number of 0.72 . The reference temperature ratio is taken to be $\phi_{0}=1 \cdot 5$, i.e. $T_{c} / T_{h}=0.5$. The constant fluid property assumption and the Boussinesq approximation are reasonable for the above $T_{c} / T_{h}$ ratio (Chang et al 1983).

We consider a base case with $\mathrm{Ra}_{E}=5 \times 10^{6}$ to demonstrate radiation affected temperature and buoyant flow fields in a square enclosure. The assumption of twodimensional laminar flow is valid for the above value of the external Rayleigh number (Larson \& Viskanta 1976; Acharya \& Goldstein 1985). The isotherm and streamline contour plots for a nonradiating fluid are shown in figure 2 for the adiabatic case and in figure 3 for the linear temperature case. The temperature and flow fields are centro-symmetric in the absence of radiation. The inner core is thermally-stratified and multicellular and is surrounded by an outer convective roll rotating in an anti-
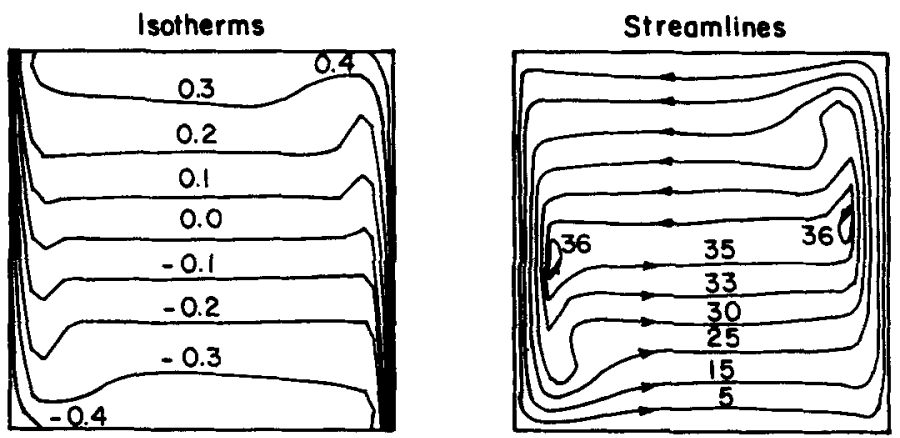

Figure 2. Isotherms and streamlines for a nonradiating fluid (adiabatic case: $\left.\operatorname{Ra}_{E}=5 \times 10^{6}\right)$. 

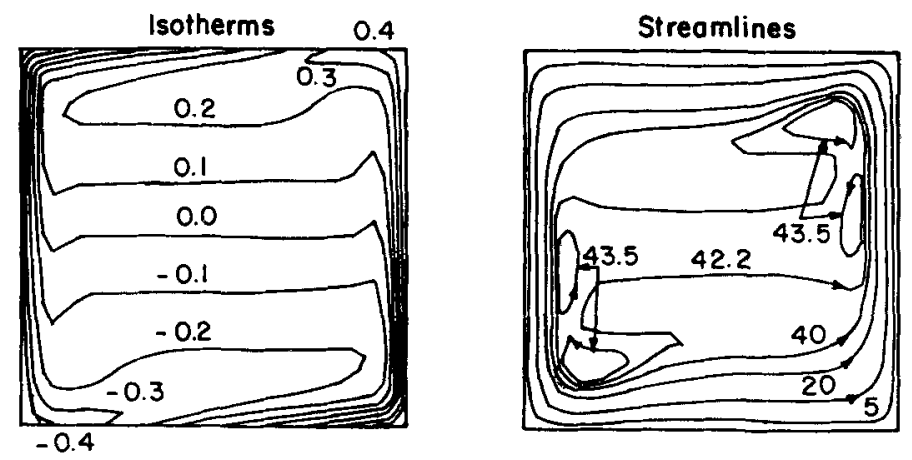

Figure 3. Isotherms and streamlines for a radiating fluid (linear temperature case: $\left.\mathrm{Ra}_{E}=5 \times 10^{6}\right)$.

clockwise sense. Large velocity and temperature gradients indicating a boundary layer structure exist in regions adjacent to the thermally active (hot and cold) walls. For the adiabatic case, the isotherms are not orthogonal to the adiabatic walls when radiation is present. Thus, while the total flux at the wall is zero, the individual contributions due to radiation and conduction are non-zero. In the case of perfectly conducting end walls strong temperature gradients are also present near the end walls. As a result, the multicellular inner core structure extends toward the upper hot and lower cold corners of the enclosure.

Radiation effects for an absorbing, emitting but nonscattering fluid $(\omega=0)$ are illustrated in figures 4 and 5 . The isotherms and streamlines presented are for an optical thickness of one and a Planck number of 0.02 which entail significant heating of the fluid by radiative transfer. The enclosure walls are assumed to be black unless otherwise noted. For the case with adiabatic end walls (figure 4), the central temperature stratification is less apparent. Temperatures above the mean temperature $T_{0}(\phi>0)$ prevail throughout most of the enclosure reflecting the penetration of the hot fluid; colder regions $(\phi<0)$ are confined to the region in the vicinity of the cold wall and the lower left part of the enclosure. The flow pattern is significantly altered due to radiation, with the most significant effect being the disappearance of the
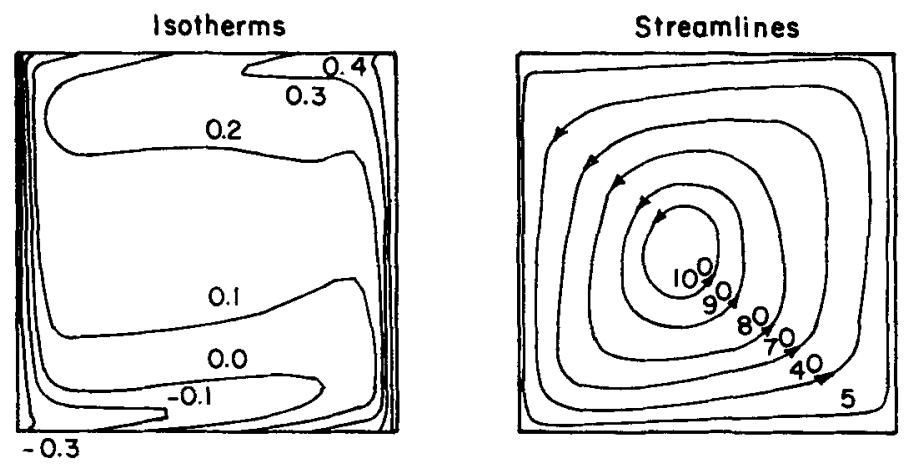

Figure 4. Isotherms and streamlines for a radiating fluid (adiabatic case: $\mathrm{Ra}_{E}=5 \times 10^{6}, \mathrm{Pl}=0.02, \tau=1 \cdot 0, \omega=0$ ). 

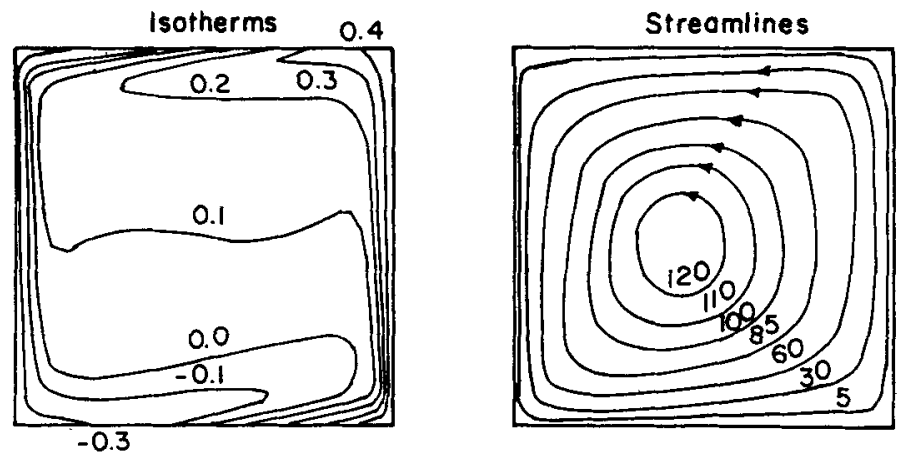

Figure 5. Isotherms and streamlines for a radiating fluid (linear temperature case: $\mathrm{Ra}_{E}=5 \times 10^{6}, \mathrm{Pl}=0.02, \tau=1 \cdot 0, \omega=0$ ).

multicellular inner core. The flow is unicellular and significantly stronger. For the perfectly conducting end walls (figure 5) the penetration of the hot wall radiation into the medium is not as large as in the adiabatic case but is still quite significant. The unicellular flow is more vigorous in this case.

Figure 6 shows the temperature variations along both end walls for the adiabatic case. For the nonradiating fluid, the temperature remains relatively uniform over the length of the walls but drops (or rises) sharply at the corners. For the radiating fluid, radiation is the dominant heat transfer mechanism. The end wall temperature variations are closer to the linear temperature condition (dashed line); as a consequence, in the presence of radiation, the temperature and flow patterns of the adiabatic and linear temperature cases (figures 4 and 5) are more similar to each other than in the nonradiating cases.

The vertical and horizontal velocity profiles along the respective horizontal and vertical midplanes are shown in figure 7 . When radiation is not accounted for, the inner core is rather stagnant. Radiation causes an overall increase in the velocities and the constant-velocity-core disappears. Large velocity gradients exist along both the thermally active walls and the end walls. As noted earlier, the flow is stronger in

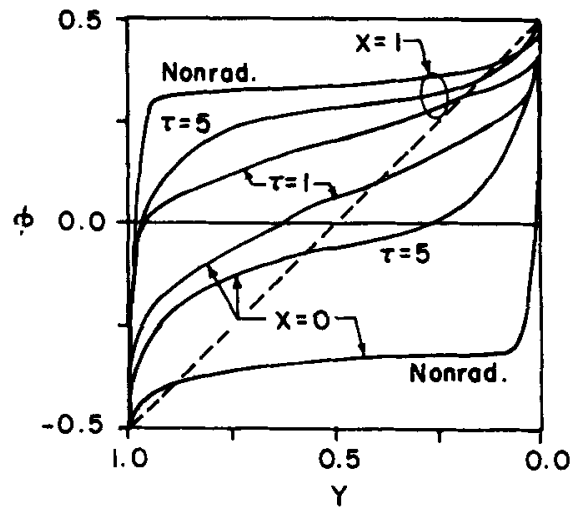

Figure 6. Temperature profiles on the end walls $(X=0$ and $X=1)$ for the adiabatic case $\mathrm{Ra}_{E}=5 \times 10^{6}, \mathrm{Pl}=0.02$, $\omega=0$. 

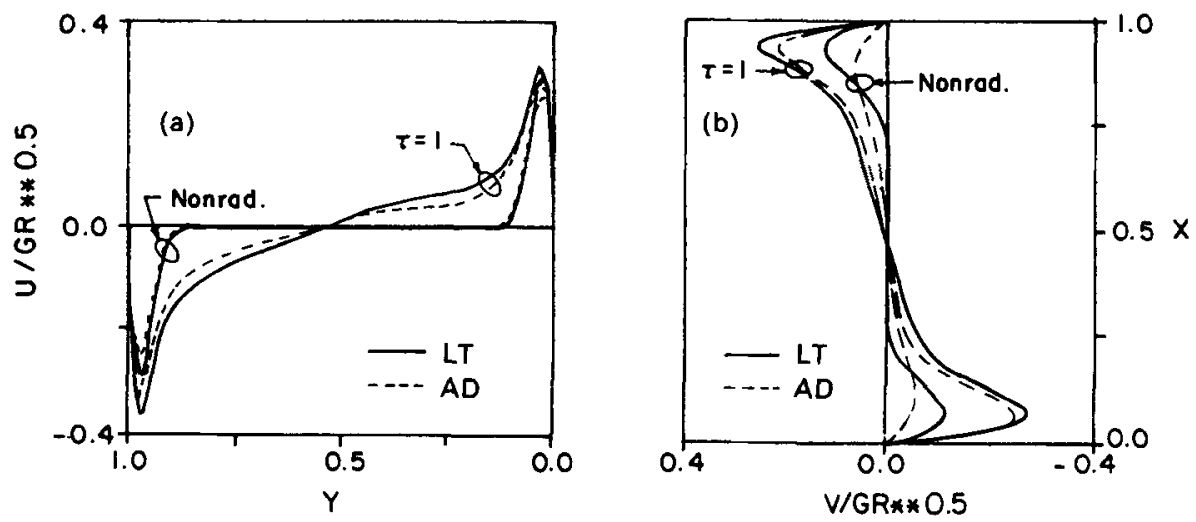

Figure 7. Velocity profiles for a radiating fluid: (a) Vertical velocity on the $X=0.5$ midplane; (b) horizontal velocity on the $Y=0.5$ midplane $\left(\operatorname{Ra}_{E}=5 \times 10^{6}\right.$, $\mathrm{Pl}=0.02, \tau=1 \cdot 0, \omega=0 ; \mathrm{AD}=$ adiabatic case, $\mathrm{LT}=$ linear temperature case).

the linear temperature case as seen by the increased magnitudes of velocities compared to the adiabatic case.

The effects of the optical thickness on the isotherm and streamline patterns are presented in figures 8 and 9 for an enclosure with perfectly conducting end walls. Figure 8 presents the temperature and flow field for an optical thickness of 5 and the general behaviour is similar to that with an optical thickness of unity. The flow is unicellular. On the other hand, for $\tau=0.2$, the radiative interactions between the enclosure walls are greater in an optically thinner medium because of lessened attenuation by the fluid. The colder regions extend further into the mid-region (figure 9) and a strong core temperature stratification manifests itself. The resulting flow exhibits a multi-cellular pattern. The inner core consists of a primary convective roll in the lower half and a secondary roll in the upper half.

Figure 10 illustrates the changes in the temperature and flow fields for an isotropically scattering fluid with $\omega=0 \cdot 5$. Compared to the nonscattering fluid case (figure 5), there is less absorption of radiation by an absorbing and scattering fluid.
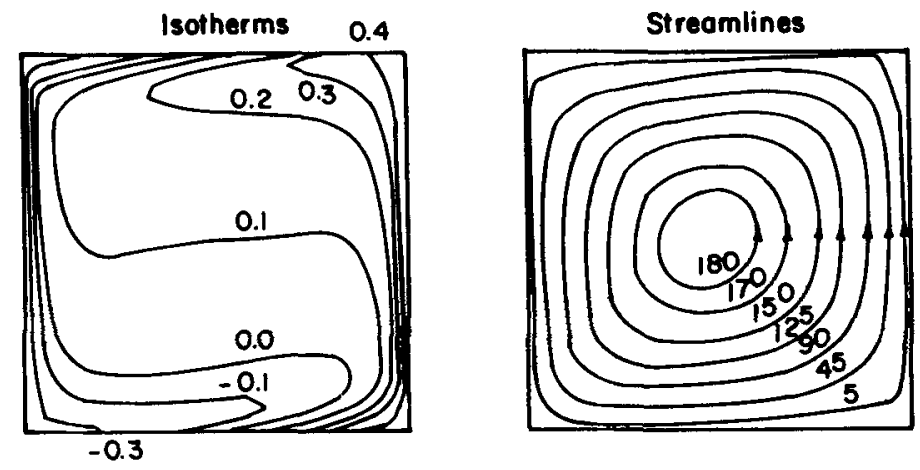

Figure 8. Isotherms and streamlines for a radiating fluid (linear temperature case: $\mathrm{Ra}_{E}=5 \times 10^{6}, \mathrm{Pl}=0.02, \tau=5 \cdot 0, \omega=0$ ). 

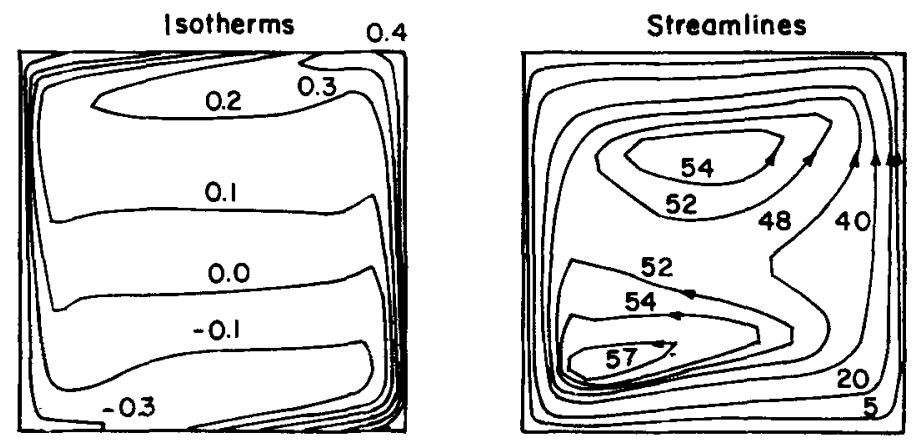

Figure 9. Isotherms and streamlines for a radiating fluid (linear temperature case: $\mathrm{Ra}_{E}=5 \times 10^{6}, \mathrm{Pl}=0 \cdot 02, \tau=0 \cdot 2, \omega=0$ ).

Therefore the temperature distribution is more similar to that for an optically thinner nonscattering fluid (figure 9). The flow pattern also exhibits two distinct rolls in the inner core surrounded by an outer wall-adjacent roll, all rotating in the counterclockwise direction. Compared to the adiabatic case (not shown) where nearly symmetrical inner rolls are obtained, the inner flow pattern is less symmetrical in the conducting end wall case.

Average values of the dimensionless total heat flux (conductive and radiative combined) and the radiative contribution to the total heat flux are presented in table 2. Overall, the heat transfer rates are much higher for a radiating fluid. For $P 1=0.02$, the net heat input (at $X=0$ and $Y=0$ ) is about $5-12 \%$ higher for the linear end-wall-temperature case than that for the adiabatic case. The ratio of the total and the radiative fluxes on the hot wall $(Y=0)$ vary little from one case to the other. Radiation accounts for around $75-80 \%$ of the total heat flux on the hot wall in all three cases $(\tau=0 \cdot 2,1 \cdot 0,5 \cdot 0)$. There is an additional but small heat input from the lower end wall $(X=0)$ in the linear temperature case. Its magnitude increases with increasing optical thickness and the corresponding radiative contribution increases from a net heat loss (output) to a net heat gain (input). The total heat output from the cold wall $(Y=1)$ is obviously identical to the total heat input from the hot wall for the adiabatic case, but is around $20 \%$ lower for the linear temperature
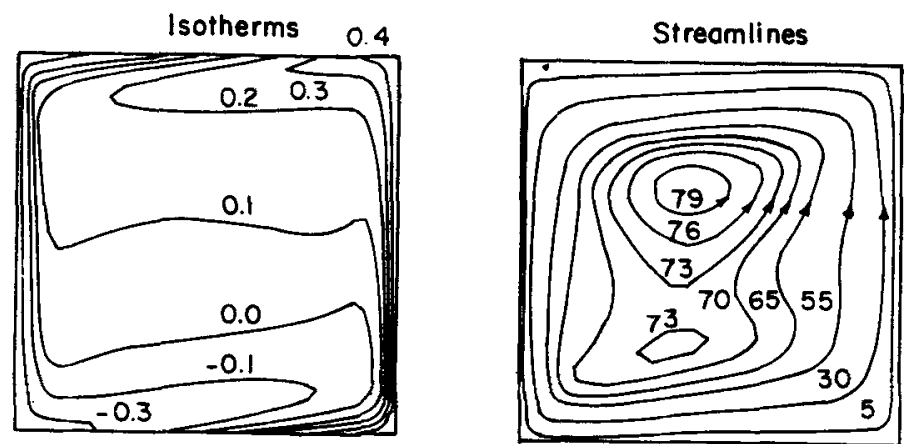

Figure 10. Isotherms and streamlines for a scattering fluid (linear temperature case: $\mathrm{Ra}_{E}=5 \times 10^{6}, \mathrm{Pl}=0.02, \tau=1 \cdot 0, \omega=0.5$ ). 
Table 2. Average heat fluxes on the enclosure walls $\left(\mathrm{Ra}_{E}=5 \times 10^{6}, \mathrm{Pl}=0.02, \omega=0\right)$.

\begin{tabular}{|c|c|c|c|c|c|c|c|c|c|}
\hline \multirow[b]{2}{*}{$\tau$} & & \multicolumn{2}{|c|}{$\begin{array}{l}\text { Hot wall } \\
(Y=0)\end{array}$} & \multicolumn{2}{|c|}{$\begin{array}{l}\text { Cold wall } \\
(Y=1)\end{array}$} & \multicolumn{2}{|c|}{$\begin{array}{l}\text { Bottom end wall } \\
\qquad(X=0)\end{array}$} & \multicolumn{2}{|c|}{$\begin{array}{l}\text { Top end wall } \\
\qquad(X=1)\end{array}$} \\
\hline & & $Q_{T^{+}}$ & $\left(Q_{R} \phi_{0} / \mathrm{Pl}\right)^{*}$ & $Q_{T}$ & $\left(Q_{R} \phi_{0} / \mathrm{Pl}\right)$ & $Q_{T}$ & $\left(Q_{R} \phi_{0} / \mathrm{Pl}\right)$ & $Q_{T}$ & $\left(Q_{R} \phi_{0} / P I\right)$ \\
\hline Nonrad. & $\begin{array}{l}\mathrm{AD}^{\mathrm{a}} \\
\mathrm{LT}^{\mathrm{b}}\end{array}$ & $\begin{array}{l}13.83 \\
10.69\end{array}$ & $\begin{array}{l}0.00 \\
0.00\end{array}$ & $\begin{array}{l}13 \cdot 83 \\
10 \cdot 69\end{array}$ & $\begin{array}{l}0.00 \\
0.00\end{array}$ & $\begin{array}{l}0.00 \\
5.28\end{array}$ & $\begin{array}{l}0.00 \\
0.00\end{array}$ & $\begin{array}{l}0.00 \\
5 \cdot 28\end{array}$ & $\begin{array}{l}0.00 \\
0.00\end{array}$ \\
\hline 0.2 & $\begin{array}{l}\text { AD } \\
\text { LT }\end{array}$ & $\begin{array}{l}46 \cdot 45 \\
47 \cdot 52\end{array}$ & $\begin{array}{l}37 \cdot 67 \\
38 \cdot 22\end{array}$ & $\begin{array}{l}46.45 \\
38.73\end{array}$ & $\begin{array}{l}31 \cdot 87 \\
27 \cdot 37\end{array}$ & $\begin{array}{l}0.00 \\
1 \cdot 14\end{array}$ & $\begin{array}{l}-5.09 \\
-3.33\end{array}$ & $\begin{array}{l}0.00 \\
9.93\end{array}$ & $\begin{array}{r}-2 \cdot 07 \\
4 \cdot 31\end{array}$ \\
\hline 1.0 & $\begin{array}{l}\mathrm{AD} \\
\mathrm{LT}\end{array}$ & $\begin{array}{l}39 \cdot 36 \\
40 \cdot 38\end{array}$ & $\begin{array}{l}31 \cdot 63 \\
32 \cdot 40\end{array}$ & $\begin{array}{l}39 \cdot 36 \\
31 \cdot 96\end{array}$ & $\begin{array}{l}24 \cdot 39 \\
20 \cdot 14\end{array}$ & $\begin{array}{l}0.00 \\
2.25\end{array}$ & $\begin{array}{l}-3 \cdot 84 \\
-1 \cdot 24\end{array}$ & $\begin{array}{r}0.00 \\
10.67\end{array}$ & $\begin{array}{r}-1 \cdot 87 \\
4 \cdot 70\end{array}$ \\
\hline $5 \cdot 0$ & $\begin{array}{l}\mathrm{AD} \\
\mathrm{LT}\end{array}$ & $\begin{array}{l}31.93 \\
31.54\end{array}$ & $\begin{array}{l}23 \cdot 77 \\
23 \cdot 78\end{array}$ & $\begin{array}{l}31.93 \\
24 \cdot 79\end{array}$ & $\begin{array}{l}16 \cdot 05 \\
12 \cdot 39\end{array}$ & $\begin{array}{l}0.00 \\
6.33\end{array}$ & $\begin{array}{r}-1.72 \\
2.98\end{array}$ & $\begin{array}{r}0.00 \\
13.08\end{array}$ & $\begin{array}{r}-0.79 \\
6.53\end{array}$ \\
\hline
\end{tabular}

${ }^{\mathrm{a}} \mathrm{AD}$ : adiabatic case; ${ }^{\mathrm{b}} \mathrm{LT}$ : linear temperature case; ${ }^{+}$dimensionless total flux; ${ }^{*}$ dimensionless radiative flux.

case. The radiative contribution to the total heat flux on the cold wall drops down to $50-65 \%$ for both cases. Approximately one-fourth of the total heat output from the enclosure (at $X=1$ and $Y=1)$ is transferred out from the top end wall $(X=1)$ for the linear temperature case; about half of this amount is by radiation.

The effect of nonblack (reflecting) end walls on the boundary layer structure along the hot and cold walls is small as can be seen in figure 11 . The radiative heat fluxes on the end walls are drastically reduced for $\varepsilon=0 \cdot 2$. The net heat input, with a small contribution from the lower end wall, remains relatively unchanged, but the total heat output from the cold wall increases to compensate for the decreased radiative heat loss from the top end wall.

\subsection{Computational issues}

The computations were performed on a Floating Point Systems 264 Attached Processor with an IBM 3084 computer as the front end machine. A typical case with
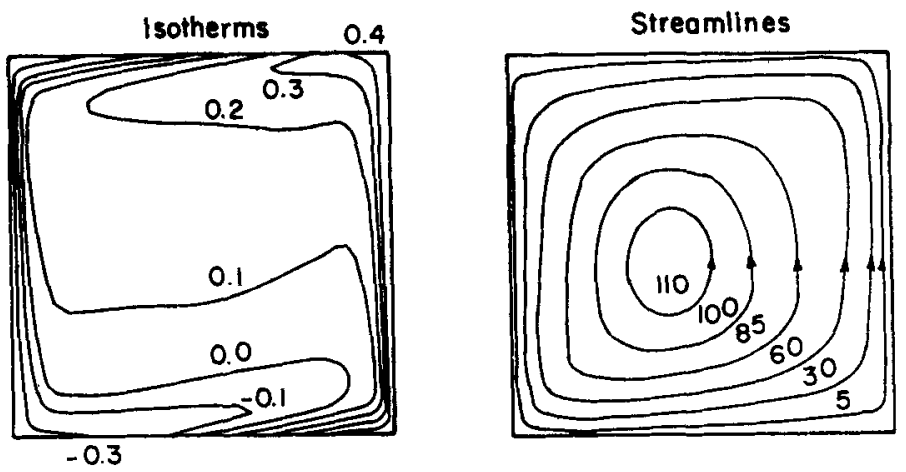

Figure 11. Isotherms and streamlines for a radiating fluid (linear temperature case with end wall emissivity of $0 \cdot 2: \mathrm{Ra}_{E}=5 \times 10^{6}, \mathrm{Pl}=0 \cdot 02, \tau=0 \cdot 2, \omega=0$ ). 
radiation using S4 quadrature required about 500 iterations $(0 \cdot 37 \mathrm{CPU}$ seconds per iteration) for the $32 \times 32$ grid. The difference in CPU times between a scattering and nonscattering case was insignificant. Although problems with scattering require inner iterations in the discrete ordinates calculations, only one inner iteration is needed per global iteration after the first $10-15$ outer iterations and as the calculations move rapidly towards convergence. This trend also holds for problems with reflective boundaries which also require inner iterations.

\section{Conclusions}

When radiation is the predominant mechanism of heat transfer, the effects of radiation on natural convection in an externally heated square enclosure with perfectly conducting end walls are similar to, but more pronounced than those in an enclosure with adiabatic end walls. The temperature distribution is significantly altered by radiation, resulting in higher temperatures in the inner region of the enclosure and, for adiabatic end walls, an end wall temperature closer to the linear temperature profile. Velocities are intensified in the presence of radiation. For the linear temperature case, the resulting flows are stronger than the corresponding flow in the adiabatic case and are generally characterized by a single prevalent convective roll. A multicellular inner core can develop for an optically thin or scattering fluid. Along the hot wall and the end walls, the heat input is larger for the conducting end wall case.

\section{List of symbols}

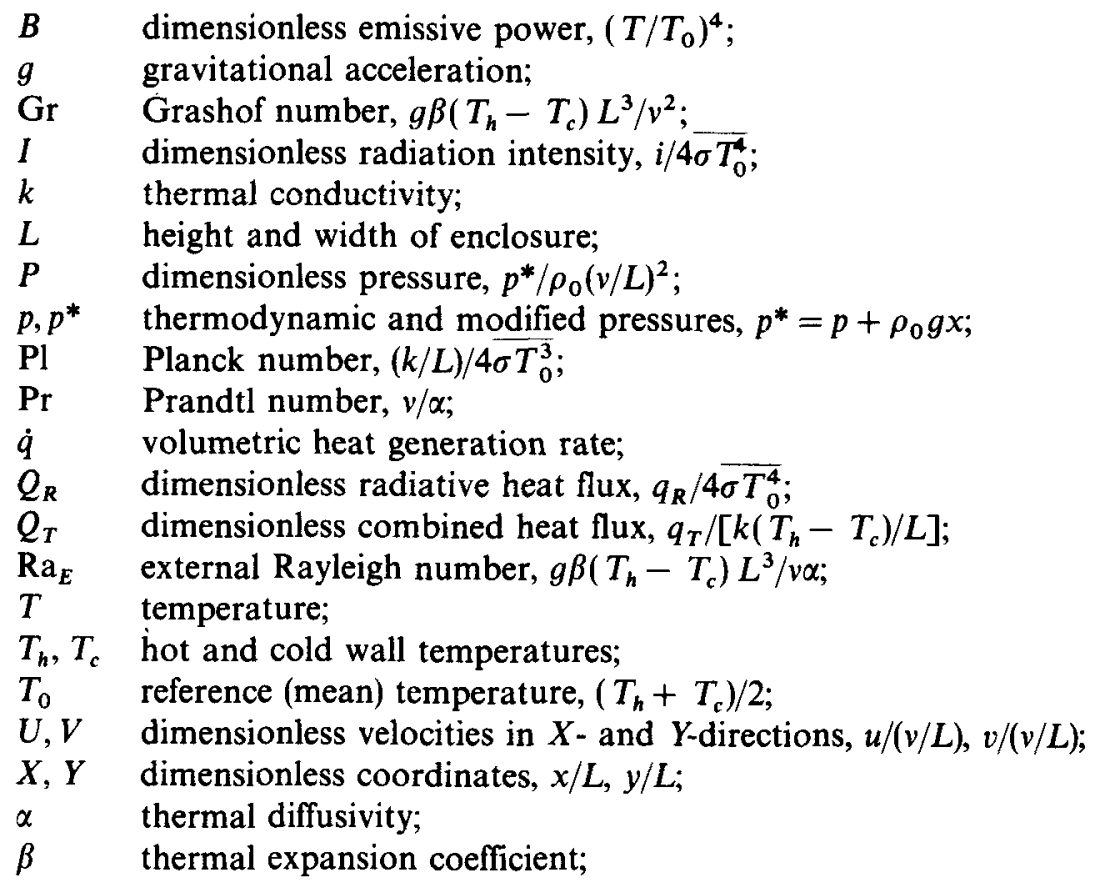


$\varepsilon \quad$ wall emissivity;

$\kappa \quad$ absorption coefficient;

$\mu, \xi \quad$ direction cosines in $X$ - and $Y$-directions;

$v \quad$ kinematic viscosity;

$\rho \quad$ density, $\rho_{0}\left[1-\beta\left(T-T_{0}\right)\right]$;

$\rho_{0} \quad$ reference density;

$\sigma \quad$ scattering coefficient;

$\bar{\sigma} \quad$ Stefan-Boltzmann constant;

$\tau \quad$ optical thickness, $(\kappa+\sigma) L$;

$\phi \quad$ dimensionless temperature, $\left(T-T_{0}\right) /\left(T_{h}-T_{c}\right)$;

$\phi_{0} \quad$ reference temperature ratio, $T_{0} /\left(T_{h}-T_{c}\right)$;

$\psi \quad$ stream function;

$\boldsymbol{\Omega}$ direction vector, $\mu \mathbf{i}+\xi \mathbf{j}$;

$\omega \quad$ scattering albedo;

$\nabla \quad$ dimensionless gradient operator, $\mathbf{i} \partial / \partial X+\mathbf{j} \partial / \partial Y$.

\section{References}

Acharya S, Goldstein R J 1985 Natural convection in an externally heated vertical or inclined square box containing internal energy sources. J. Heat Transfer 107: 855-865

Chang L C, Yang K T, Lloyd J R 1983 Radiation-natural convection interactions in twodimensional complex enclosures. J. Heat Transfer 105: 89-95

Desrayaud G, Lauriat G 1985 Natural convection of a radiating fluid in a vertical layer. $J$. Heat Transfer 107: 710-712

Duderstadt J J, Martin W R 1979 Transport theory (New York: Wiley)

ElSherbiny S M, Hollands K G T, Raithby G D 1982 Effect of thermal boundary conditions on natural convection in vertical and inclined air layers. J. Heat Transfer 104: 515-520

Fiveland W A 1987 Three-dimensional radiative heat transfer solutions by the discrete-ordinates method. Fundamentals and applications of radiation heat transfer (eds) A M Smith, T F Smith (New York: ASME) HTD-72

Fusegi T, Farouk B 1986 Radiation-convection interactions in asymmetrically heated square enclosures. Numerical methods in heat transfer (eds) J L S Chen, K Vafai (New York: ASME) HTD-62

Fusegi T, Farouk B 1987 Radiation-convection interactions of a non-gray gas in a square enclosure. Heat and mass transfer in fire (eds) A K Kulkarni, Y Jaluria (New York: ASME) HTD-73

Kassemi M, Duval W M B 1990 Interactions of surface radiation with convection in crystal growth by vapor transport. J. Thermophys. Heat Transfer 4: 605-611

Kassemi M, Naraghi M H N 1990 Analysis of radiation-natural convection interactions in 1-G and low-G environments using the discrete exchange factor method. $A I A A / A S M E$ Thermophysics Conference, Seattle

Larson D W, Viskanta R 1976 Transient combined laminar free convection and radiation in a rectangular enclosure. J. Fluid Mech. 78: 65-85

Lauriat $\mathrm{G}$ 1982a Combined radiation-convection in gray fluids enclosed in vertical cavities. J. Heat Transfer 104: 609

Lauriat $G 1982 \mathrm{~b}$ Numerical study of the interaction of natural convection with radiation in nongray gases in a narrow vertical cavity. Heat Transfer, Proc. of the 1982 Int. Conf. (Washington, DC: Hemisphere) pp. 153-160

Naraghi M H N, Kassemi M 1989 Radiative transfer in a rectangular enclosure; A discretized exchange factor solution. J. Heat Transfer 117: 1117-1124

Ostrach S 1988 Natural convection in enclosures. J. Heat Transfer 110: 1175-1190

Patankar S V 1980 Numerical heat transfer and fluid flow (New York: Hemisphere) 
Tan $\mathrm{Z} 1989$ Radiative heat transfer in multidimensional emitting, absorbing and anisotropic scattering media - mathematical formulation and numerical method. J. Heat Transfer 111: $141-147$

Tan Z, Howell J R 1991 Combined radiation and natural convection in a square enclosure with participating medium. Int. J. Heat Mass Transfer 34: 79-97

Viskanta R 1984 Radiation heat transfer. Fortschritte Der Verfahrenstechnik 22: 51-81

Viskanta R, Menguc M P 1987 Radiation heat transfer in combustion systems. Prog. Energy Combust. Sci. 13: 97-160

Webb B W, Viskanta R 1987 Radiation-induced buoyancy driven flow in rectangular enclosures: Experiment and analysis. J. Heat Transfer 109: 427-433

Yang K T 1986 Numerical modeling of natural convection-radiation interactions in enclosures. Heat Transfer. Proc. of the 1986 Int. Conf.(eds) C L Tien, V P Carey, J K Ferrell (Washington, DC: Hemisphere) vol. 1, pp. 131-140

Yücel A, Acharya S, Williams M L 1989 Natural convection and radiation in a square enclosure. Numer. Heat Transfer A15: 261-278 